\title{
Alterations of the thrombin generation profile in rheumatoid arthritis
}

\author{
Anita Kern ${ }^{1,2}$ - Attila Balog ${ }^{3}$ Sonja Dulic ${ }^{3}$ - Eszter Barabás ${ }^{1}$ - Mária Kiszelák ${ }^{2}$ • \\ Barna Vásárhelyi ${ }^{1}$
}

Published online: 19 July 2015

(c) Springer Science+Business Media New York 2015

\begin{abstract}
Rheumatoid arthritis (RA) is a chronic inflammatory autoimmune disorder resulting in the erosion of the cartilage and bone. Systemic involvement including the cardiovascular system with the risk of atherosclerosis may also occur. Calibrated automated thrombogram (CAT), a commercially available thrombin generation assay is suitable for the general assessment of the functionality of coagulation system. In this study we performed CAT assay in RA patients and in non-affected control subjects (matched for age, sex and comorbidities). Among the CAT parameters Velocity Index increased (from 60 to $83 \mathrm{nM}$ / min), Lag Time and Time to Peak decreased (from 3.47 to $2.83 \mathrm{~min}$ and from 6.98 to 5.58 min respectively) in RA. On the other hand, Endogenous Thrombin Potential values decreased (from 1242 to $1108 \mathrm{nM}$ min). The observed alterations were not associated with the applied therapy. These results indicate that the velocity of thrombin formation is increased, while the thrombin generating capability is reduced in RA.
\end{abstract}

Keywords Thrombin generation - Rheumatoid arthritis · Thrombosis · Biological therapy

Anita Kern

kern.anita1@gmail.com; akern@diagon.com

1 Department of Laboratory Medicine, Faculty of Medicine, Semmelweis University, Nagyvárad tér 4, 1089 Budapest, Hungary

2 Diagon Ltd., Baross utca 48-52, 1047 Budapest, Hungary

3 Department of Rheumatology, Faculty of Medicine, Albert Szent-Györgyi Health Center, University of Szeged, Kálvária sgt. 57, 6725 Szeged, Hungary

$\begin{array}{ll}\text { Abbreviations } \\ \text { aPTT } & \text { Activated partial thromboplastin time } \\ \text { CAT } & \text { Calibrated Automated Thrombogram } \\ \text { CRP } & \text { C reactive protein } \\ \text { DAS } & \text { Disease activity score } \\ \text { DMARD } & \text { Disease modifying anti-rheumatic drugs } \\ \text { ESR } & \text { Erythrocyte sedimentation rate } \\ \text { ETP } & \text { Endogenous thrombin potential } \\ \text { NSAID } & \text { Non-steroidal anti-inflammatory drugs } \\ \text { PT } & \text { Prothrombin time } \\ \text { RA } & \text { Rheumatoid arthritis } \\ \text { RF } & \text { Rheumatoid factor } \\ \text { TG } & \text { Thrombin generation } \\ \text { TT } & \text { Thrombin time } \\ \text { v index } & \text { Velocity Index }\end{array}$

\section{Introduction}

Rheumatoid arthritis (RA) is a chronic inflammatory autoimmune disorder with a symmetrical small-joint polyarthritis, but extra-articular manifestations may be present both at disease onset and in established disease. There are a number of studies indicating the augmented risk of cardiovascular diseases in RA including atherothrombosis $[1,2]$ and venous thromboembolism [3, 4].However, from the limited data available it is still nebulous whether the increased risk is inherent with RA itself or is partly due to potential confounders [3]. Indeed, in addition to the thrombogenic effects of systemic inflammation, immobilisation, plasma hyperviscosity [5], disturbed venous blood flow [6] and adverse effects of anti-rheumatic agents [7] may also contribute to the thrombotic tendency in RA.

Beside standard coagulation tests [prothrombin time (PT), activated partial thromboplastin time (aPTT), 
thrombin time (TT) and fibrinogen] novel biomarkers in the assessment of thrombotic potential have been recently introduced into the laboratory diagnostic arsenal. Thrombin generation (TG) test, a novel global haemostasis assay, is a tool for the general functional assessment of haemostasis. The result of this test reflects reliably thrombotic or bleeding potential that can be triggered by physiologic or iatrogenic stimuli [8]. During TG test coagulation is provoked in the plasma sample by the addition of a small amount of triggering agent (usually thromboplastin). The activity of thrombin is monitored during the entire coagulation process and graphically represented on the thrombogram (Fig. 1). TG profile is characterised by descriptive parameters of the TG curve: Endogenous Thrombin Potential (ETP, total thrombin generating potential of the sample), Peak (maximum thrombin activity), Lag Time (initiation time to thrombin burst), Time to Peak (time to maximum thrombin activity) and Velocity Index ( $\mathrm{v}$ index, slope of the thrombin burst).

Albeit TG measurements are still not standardized, commercially available tests are already in use. Calibrated automated thrombogram (CAT) is one of the most widely used TG assays. CAT is increasingly used to assess thrombotic risk in a number of disorders including cancer [9], or anticoagulant treatment [10].

Until now only two studies assessed TG in RA. Undas et al. however, collected indirect data regarding TG; they developed an in silico model and estimated TG profile by analysing a number of distinct soluble pro- and anticoagulant factors in patients [11]. Their results indicate delayed but augmented thrombin burst in RA samples. However, this study was inherent with several limitations (e.g. the control group consisted of healthy subjects instead of nonaffected controls and study population was heterogeneous in term of age and gender). In a more recent study Prati et al. empirically determined the TG profiles in RA [12]. They detected alteration in all TG parameters suggesting a reduced TG in RA (indicative for an increased bleeding

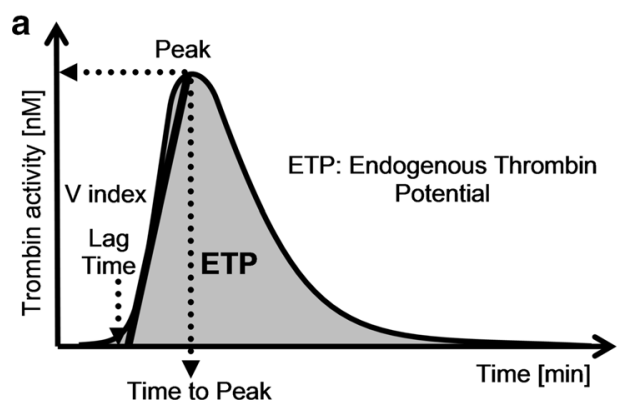

Fig. 1 Thrombogram, graphical representation of a thrombin generation (TG) measurement. a Parameters of thrombogram. b Characteristic alterations of thrombogram in thrombophilia and haemophilia. Augmented peak, ETP and $\mathrm{v}$ index, and decreased Lag Time and tendency), but no data about the study population and the applied method are provided.

In this study we aimed to evaluate TG parameters in a well-defined and homogeneous RA population and investigated the impact of RA and applied therapy on measured values.

\section{Patients and methods}

\section{Patients}

Twenty-four and 25 postmenopausal women with and without RA, respectively, matched for age and comorbidities were included in the study. RA patients and control subjects (with joint complaints, but no inflammatory condition) were recruited from the same department. Exclusion criteria were oral anticoagulant therapy or heparin therapy. Patients were treated with corticosteroids $(n=14$, $58.3 \%$ ), nonsteroidal anti-inflammatory drugs (NSAIDs, $\mathrm{n}=5,20.8 \%)$, biologic agents $(\mathrm{n}=10,41.7 \%)$, disease modifying antirheumatic drugs (DMARDs, $\mathrm{n}=22$, $91.7 \%)$ and statins $(\mathrm{n}=5,20.8 \%)$. None of the control subjects were treated with corticosteroids, DMARDs and biologic agents. Disease activity score (DAS-28) was calculated for all patients at the time of sampling. Level of standard inflammatory markers $[\mathrm{C}$ reactive protein (CRP), erythrocyte sedimentation rate (ESR)] and that of rheumatoid factor (RF) and anti mutated citrullinate vimentin (aMCV) were measured parallel (Table 1).

Patients and subjects gave written informed consent to participate in the study. The study adhered to the tenets of declaration of Helsinki.

\section{Methods}

Venous blood samples were drawn in Vacutainer ${ }^{\circledR}$ tubes (Becton-Dickinson, USA) containing $3.2 \%$ sodium

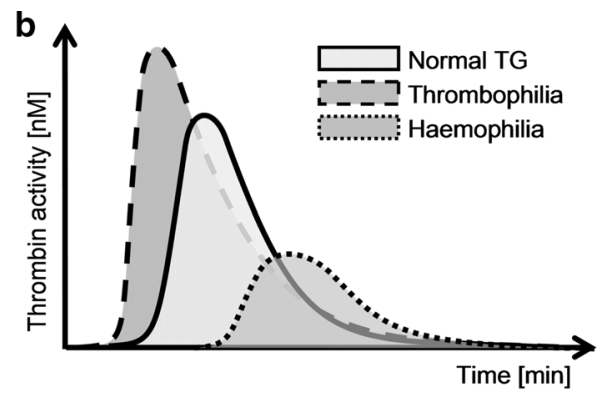

Time to Peak suggest an increase in thrombotic tendency, while reduced peak, ETP and $\mathrm{v}$ index, and prolonged Lag Time and Time to Peak indicate an increase in bleeding tendency 
Table 1 Clinical characteristics of patients with RA and controls studied

\begin{tabular}{lll}
\hline & Patient & Control \\
\hline Number & 24 & 25 \\
Age (years) & $61.7 \pm 7.5$ & $56.5 \pm 10.8$ \\
Duration of RA (years) & $11.8 \pm 8.8$ & - \\
DAS-28 & $3.29 \pm 1.41$ & - \\
CRP (mg/l) & $5.0(3.2-11.4)(\mathrm{n}=23)$ & $2.3(0-4.5)$ \\
ESR (mm/h) & $26(12-52)(\mathrm{n}=21)$ & - \\
RF (IU/ml) & $101.4(34.9-404.3)(\mathrm{n}=21)$ & - \\
aMCV & $19(79.2 \%)$ & - \\
Diabetes mellitus & $3(12.5 \%)$ & $3(12.0 \%)$ \\
Hypothyreosis & $6(25.0 \%)$ & $4(16.0 \%)$ \\
Hypertension & $14(58.3 \%)$ & $13(52.0 \%)$ \\
Osteoporosis & $5(20.8 \%)$ & $5(20.0 \%)$ \\
Hypercholesterolemia & $6(25.0 \%)$ & - \\
Coxathrosis & - & $6(24.0 \%)$ \\
Gonarthrosis & - & $6(24.0 \%)$ \\
Spondylosis & - & $15(60.0 \%)$ \\
\hline
\end{tabular}

Qualitative data are expressed as number (\%) and quantitative data as mean \pm standard deviation or median (1st-3rd interquartile), as appropriate

$a M C V$ anti mutated citrullinate vimentin, $C R P \mathrm{C}$ reactive protein, $D A S-28$ disease activity score, ESR erythrocyte sedimentation rate, $R A$ rheumatoid arthritis, $R F$ rheumatoid factor

citrate. For standard coagulation tests (see below) the samples were centrifuged at $2500 \times g$ for $10 \mathrm{~min}$. To obtain platelet poor plasma (PPP) for the TG measurement the samples were centrifuged first at $2500 \times g$ for $10 \mathrm{~min}$, then at $2500 \times \mathrm{g}$ for $20 \mathrm{~min}$. Samples were immediately frozen and stored at $-80{ }^{\circ} \mathrm{C}$ until measurements (done within 1 month).

Standard coagulation parameters (PT, aPTT, TT and fibrinogen) were determined with reagents from Diagon (Hungary) on Coag XL automated coagulometer (Diagon, Hungary).

CAT method (Diagnostica Stago, France) was performed by applying PPP Reagent (Thrombinoscope, The Netherlands). Briefly, $80 \mu \mathrm{l}$ plasma sample was mixed with $20 \mu \mathrm{l}$ trigger (PPP Reagent, Thrombinoscope, The Netherlands) in 96 round bottom wells micro plate (Immulon 2HB, Thermo Fisher Scientific, USA). The measurement was started by the addition of $40 \mu \mathrm{l}$ mixture containing $\mathrm{CaCl}_{2}$ and fluorogenic substrate (FluCA-kit, Thrombinoscope, The Netherlands). All samples were run in duplicate at $37{ }^{\circ} \mathrm{C}$. Each plasma sample was calibrated individually by applying Thrombin Calibrator (Thrombinoscope, The Netherlands) in order to correct the fluorescent signal for inner filter effect, substrate consumption and variability in colour of plasma. Fluorescence was measured with Fluoroskan Ascent (Thermo Fisher Scientific, USA) reader applying $390 \mathrm{~nm}$ excitation and $460 \mathrm{~nm}$ emission filters. TG parameters were calculated with Thrombinoscope Software (Thrombinoscope, The Netherlands). The following TG parameters were determined: Endogenous Thrombin Potential (ETP, nM min), Peak (nM), Lag Time (min), Time to Peak (min) and Velocity Index ( $v$ index, $\mathrm{nM} / \mathrm{min})$.

\section{Statistical analysis}

Normal distribution of data was analysed with AndersonDarling normality test. Qualitative data are expressed as number $(\%)$ and quantitative data as mean \pm standard deviation or median (1st-3rd interquartile), as appropriate. Coagulation parameters were compared between RA and non-affected control group, and between RA patients receiving standard, or standard and biological therapy. Comparison between groups was performed with Student t-test in case of normal distribution or with MannWhitney $U$ test in case of non-normal distribution. Coagulation parameters of RA patients were correlated with age, disease duration, DAS-28, CRP, ESR and RF. Correlations were assessed with Pearson or Spearman rank coefficient, as appropriate. All tests were two sided, and $\mathrm{p}$ values $<0.05$ were considered statistically significant. Statistical analyses were performed with Minitab ${ }^{\circledR}$ Release 14.1.

\section{Results}

Twenty-four patients and 25 control subjects were enrolled into the study. RA patients had significantly higher TT, although the values were within the normal range and the difference was clinically irrelevant. PT, aPTT and fibrinogen levels were not statistically different. On the other hand, several CAT parameters differed significantly between RA and control group; Lag Time, Time to Peak and ETP were lower, while $\mathrm{v}$ index were higher in RA than in control group (Table 2).

None of the standard coagulation parameters were associated with age, RA duration, RF, aMCV or ESR values in the RA group. Within RA group, fibrinogen levels correlated with DAS-28 $(r=0.680, p<0.0005)$ and with CRP $(\rho=0.663, p=0.001)$. DAS-28 also correlated with Lag Time $(\rho=0.438, p=0.032)$ and $v$ index ( $\rho=0.526, p=0.008)$. No difference was observed in RA subgroups receiving DMARDs and those on combination with DMARD and biologic agent. 
Table 2 Comparison of standard coagulation tests and CAT parameters of rheumatoid arthritis (RA) and control group, and that of patients on DMARD—biologic therapy and those receiving DMARDs only

\begin{tabular}{|c|c|c|c|c|c|c|}
\hline & RA & Control & $\begin{array}{l}\mathrm{p} \\
\text { RA versus } \\
\text { control }\end{array}$ & $\begin{array}{l}\text { Biologic } \\
\text { agents+ }\end{array}$ & Biologic agents- & $\begin{array}{l}\mathrm{p} \\
\text { Biologic agents }+ \text { versus } \\
\text { biologic agents- }\end{array}$ \\
\hline Number & 24 & 25 & & 10 & 14 & \\
\hline PT (INR) & $1.05 \pm 0.05$ & $1.06 \pm 0.05$ & ns. & $1.05 \pm 0.04$ & $1.03(1.00-1.09)$ & ns. \\
\hline aPTT (s) & $35.3(33.9-39.8)$ & $34.3 \pm 3.3$ & ns. & $38.1 \pm 5.9$ & $34.7(33.6-36.7)$ & ns. \\
\hline TT (s) & $18.8 \pm 1.2$ & $17.7 \pm 1.2$ & 0.003 & $18.7 \pm 1.2$ & $18.8(18.1-19.2)$ & ns. \\
\hline Fibrinogen $(\mathrm{g} / \mathrm{l})$ & $3.29 \pm 0.56$ & $3.09 \pm 0.51$ & ns. & $3.13 \pm 0.57$ & $3.41 \pm 0.55$ & ns. \\
\hline ETP (nM min) & $1108 \pm 168$ & $1242 \pm 186$ & 0.012 & $1157 \pm 196$ & $1074 \pm 142$ & ns. \\
\hline Peak (nM) & $236 \pm 44$ & $209 \pm 60$ & ns. & $234 \pm 53$ & $226(208-238)$ & ns. \\
\hline Lag Time (min) & $2.83(2.67-3.25)$ & $3.47 \pm 0.91$ & $\mathbf{0 . 0 3 9}$ & $2.72 \pm 0.33$ & $3.12 \pm 0.69$ & ns. \\
\hline Time to Peak (min) & $5.58 \pm 0.79$ & $6.98 \pm 1.67$ & 0.001 & $5.35 \pm 0.70$ & $5.74 \pm 0.83$ & ns. \\
\hline $\mathrm{v}$ index $(\mathrm{nM} / \mathrm{min})$ & $83(72-121)$ & $60(35-104)$ & 0.017 & $98 \pm 35$ & $79(73-106)$ & ns. \\
\hline
\end{tabular}

Data with normal distribution are expressed as mean \pm standard deviation and in case of non-normal distribution as median (1st-3rd interquartile)

Significant differences are marked with bold letters

aPTT activated partial thromboplastin time, ETP endogenous thrombin potential, INR international normalised ratio, $P T$ prothrombin time, $T T$ thrombin time, $v$ index Velocity Index

\section{Discussion}

In this study characteristic alterations were demonstrated in TG parameters of RA patients compared to those in the control subjects. The majority of routine tests of hemostasis (except for fibrinogen level) were normal in RA.

CAT measurement as a global haemostasis assay for the assessment of TG provides a comprehensive tool for the assessment of functional state of the haemostatic system; it reflects the contribution of both pro- and anticoagulant processes to the haemostatic balance. As a result CAT can be considered a more sensitive method than routine coagulation tests to describe the thrombotic state of patients in complex haemostatic alterations such as chronic systemic inflammation in RA.

Among the 5 measured CAT parameters (ETP, Peak, Lag Time, Time to Peak, v index) 4 differed significantly in RA from those in the control group. The characteristics of CAT curve was not clearly specific for thrombophilia or haemophilia, as its parameters shared a mixed pattern: kinetic CAT parameters (i.e. reduced Lag Time and Time to Peak and increased $\mathrm{v}$ index) suggested an increase in general thrombotic tendency, while reduced ETP indicated an impaired thrombotic potential. Typical examples for TG curves of a RA patient and a control subject are represented on Fig. 2. The net effect of these changes, however, was an earlier and accelerated formation of reduced amount of thrombin. These changes were associated with RA activity (i.e. DAS-28 scores).

The mutual relationship between inflammation and coagulation is well established. While inflammatory mechanisms shift haemostatic balance towards increased coagulation, coagulation processes can enhance inflammatory response [reviewed in 5, 13]. Inflammation promotes procoagulation and downregulates anticoagulant processes by increasing platelet count and reactivity, elevating fibrinogen and CRP concentrations, inducing expression of protease activated receptors on endothelium, and tissue factor expression on cell surface of leucocytes. (Indeed, in RA patients the determined fibrinogen levels correlated with CRP levels and DAS-28.) Elevated CRP level promotes interactions of monocytes and endothelial cells [14], and formation of plasminogen activator inhibitor-1 [15] and that of tissue factor [16]. Inflammatory mediators affect anticoagulant pathways by the downregulation of thrombomodulin and endothelial protein $\mathrm{C}$

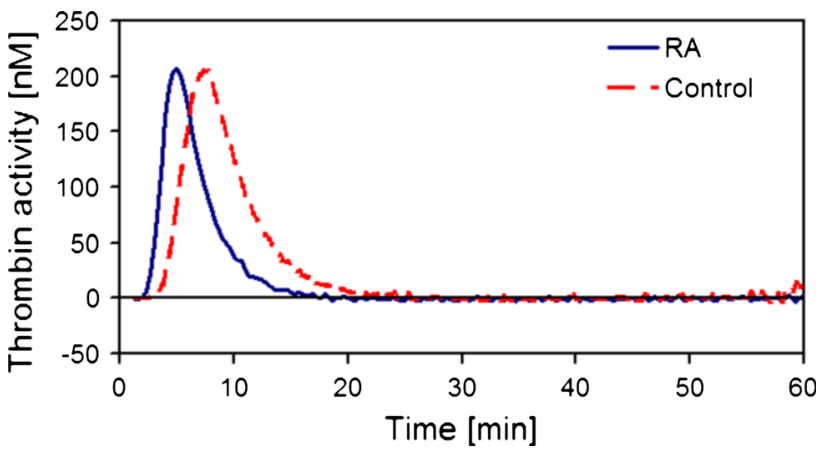

Fig. 2 Typical thrombin generation curve of a rheumatoid arthritis (RA) patient (solid line) and that of a control subject (dashed line). In RA Endogenous Thrombin Potential, Lag Time and Time to Peak is decreased, while Velocity Index is increased 
receptor, thus diminishing protein $\mathrm{C}$ activation. In inflammation, lowered antithrombin level can be caused by reduced synthesis, degradation and consumption and the decreased synthesis of glycosaminoglycans. Lowered antithrombin activity results in delayed inhibition of the coagulation enzymes that favours thrombotic tendency.

Our observations are not fully in line with the results obtained in a recent study of Undas et al. [11]. This team analysed the TG profile of RA patients calculated with a computational model from their plasma composition. They found that compared to control group RA patients had significantly higher Peak and $\mathrm{v}$ index, and prolonged Lag Time and Time to Peak. In contrary to our results, these data indicate delayed but augmented thrombin burst in patient samples.

These contradictions are probably due to different study design, populations tested and methods used. Firstly, there are numerous differences in patient and control group selection. In the previous study the investigated population was heterogeneous in term of age, disease duration and gender, and control group consisted of healthy subjects. Our study focused on the TG profile of postmenopausal RA women, a specific subpopulation, and-to avoid the bias inherent with the use of different age-group-we enrolled age-matched control subjects with a comparable overall health status (without RA). Secondly, Undas et al. determined the TG profile by kinetic modelling, while we measured them empirically. Despite the advances of the kinetic modelling of TG, the limitations of computational modelling should be considered when calculated results are compared to measured parameters [17].

When evaluating the results of our study one should consider that each RA patient participating received DMARD agents combined with or without biological therapy. Theoretically, these agents may also influence directly (through their impact on pro- and anticoagulation factors) and indirectly (through their anti-inflammatory action) the haemostasis. We speculate that these drugs may be responsible that ETP and TT values in our RA patients were inconsistent with kinetic CAT results (they indicated an impairment in TG in RA). The detailed analysis of association between coagulation parameters, CAT parameters and drugs used excluded the contribution of biological therapies to observed alterations. (Literary data support no significant impact of biological agents on thrombotic tendency either [12].)

However, as nearly all RA patients (22/24) were given DMARD for several years, the modulatory effect of DMARD in coagulation system in RA cannot be excluded. Indeed, Undas et al. [11] demonstrated that patients taking methotrexate (a DMARD) had decreased thrombotic tendency. In our population with chronic RA requiring continuous DMARD therapy the possible impact of DMARD agents, therefore, cannot be excluded.
One should also note that our results characterize coagulation in a specific RA subpopulation homogeneous in term of gender (women) and age (postmenopause) and the long term duration of the disease. RA, however, may also affect men and patients of other ages. The possibility that haemostatic conditions in other RA subpopulation are different cannot be excluded and should be the subject for further studies including patients with higher disease activity or patients with early RA.

In conclusion, to our knowledge, we are the first to perform CAT measurements in a homogenous RA population. Our results indicate earlier and accelerated formation of a reduced amount of thrombin. This suggests that RA is associated with alterations of the haemostatic system in a way that it is prepared for fast response to provoking stimuli, thus the haemostatic balance is altered towards thrombotic tendency, but impaired thrombin amount counterbalance this feature.

\section{References}

1. Aksu K, Donmez A, Keser G (2012) Inflammation-induced thrombosis: mechanisms, disease associations and management. Curr Pharm Des 18:1478-1493

2. Gasparyan AY, Ayvazyan L, Cocco G, Kitas GD (2012) Adverse cardiovascular effects of antirheumatic drugs: implications for clinical practice and research. Curr Pharm Des 18:1543-1555

3. Ungprasert P, Srivali N, Spanuchart I, Thongprayoon C, Knight EL (2014) Risk of venous thromboembolism in patients with rheumatoid arthritis: a systematic review and meta-analysis. Clin Rheumatol 33:297-304

4. Yusuf HR, Hooper WC, Grosse SD, Parker CS, Boulet SL, Ortel TL (2015) Risk of venous thromboembolism occurrence among adults with selected autoimmune diseases: a study among a U.S. cohort of commercial. Thromb Res 135:50-57

5. Hoppe B, Dörner T (2012) Coagulation and the fibrin network in rheumatic disease: a role beyond haemostasis. Nat Rev Rheumatol 8:738-746

6. McLaughlin GE (2002) Sudden death in rheumatoid arthritis: pulmonary embolism-a fatal complication of iliopsoas bursitis. J Clin Rheumatol 8:208-211

7. Bessissow T, Renard M, Hoffman I, Vermeire S, Rutgeerts P, Van Assche G (2012) Review article: non-malignant haematological complications of anti-tumour necrosis factor alpha therapy. Aliment Pharmacol Ther 36:312-323

8. Hemker HC, Al Dieri R, De Smedt E, Béguin S (2006) Thrombin generation, a function test of the haemostaticthrombotic system. J Thromb Haemost 96:553-561

9. Panova-Noeva M, Marchetti M, Spronk HM, Russo L, Diani E, Finazzi G, Salmoiraghi S, Rambaldi A, Barbui T, Ten Cate H, Falanga A (2011) Platelet-induced thrombin generation by the calibrated automated thrombogram assay is increased in patients with essential thrombocythemia and polycythemia vera. Am J Hematol 86:337-342

10. Tripodi A, Legnani C, Chantarangkul V, Cosmi B, Palareti G, Mannucci PM (2008) High thrombin generation measured in the presence of thrombomodulin is associated with an increased risk of recurrent venous thromboembolism. J Thromb Haemost 6:1327-1333 
11. Undas A, Gissel M, Kwasny-Krochin B, Gluszko P, Mann KG, Brummel-Ziedins KE (2010) Thrombin generation in rheumatoid arthritis: dependence on plasma factor composition. Thromb Haemost 104:224-230

12. Prati C, Racadot E, Cédoz JP, Toussirot É, Wendling D (2012) Thrombin generation in rheumatoid arthritis. Joint Bone Spine 79:522-523

13. Esmon CT (2005) The interactions between inflammation and coagulation. Br J Haematol 131:417-430

14. Han KH, Hong KH, Park JH, Ko J, Kang DH, Choi KJ, Hong MK, Park SW, Park SJ (2004) C-reactive protein promotes monocyte chemoattractant protein-1-mediated chemotaxis through upregulating $\mathrm{CC}$ chemokine receptor 2 expression in human monocytes. Circulation 109:2566-2571

15. Devaraj S, Xu DY, Jialal I (2003) C-reactive protein increases plasminogen activator inhibitor-1 expression and activity in human aortic endothelial cells. Circulation 107:398-404

16. Cermak J, Key NS, Bach RR, Balla J, Jacob HS, Vercellotti GM (1993) C-reactive protein induces human peripheral blood monocytes to synthesize tissue factor. Blood 82:513-520

17. Hemker HC, Kerdelo S, Kremers RMW (2012) Is there a value in kinetic modeling of thrombin generation? No (unless...). J Thromb Haemost 10:1470-1477 